\title{
Einleitung zum Teil 2
}

Die Ausführungen in Teil 1 (Kapitel 1-3) zielten darauf ab, jenen Rahmen abzustecken, in dem die in der vorliegenden Arbeit untersuchten Dokumente mutmasslich angefertigt worden sind. Dabei standen in einem ersten Schritt (Kapitel 1) allgemeine Überlegungen zum Gegenstand dieser Arbeit im Vordergrund (Abgrenzung der Forschungsfrage, kodikologische Charakterisierung der untersuchten Dokumente, allgemeine thematische Zuordnung). Kapitel 2 beleuchtete sodann die historischen Entwicklungen zwischen etwa 1250 und 180o, wobei gerade die Zeit zwischen 1250 und 1500 und die Gegebenheiten im Städtedreieck zwischen Konya, Tabriz und Bagdad interessierten. Besondere Aufmerksamkeit wurde sodann (Kapitel 3) den sozio-politischen Verhältnissen in den betroffenen Regionen geschenkt. Im Zentrum der Aufmerksamkeit standen Männerbünde unterschiedlichster Art (Derwischorden, Futuwwa- und AhiBünde, Handwerksgilden). Auch in der GulätSchia vertretene Standpunkte wurden wiederholt beleuchtet.

Dieser Teil 2 (v.a. Kapitel 4-5, aber auch Kapitel 6 und 7) stellt nun die bedeutendsten Dokumente in Rollenform vor, die zwischen dem 14. und 19. Jh. entstanden sind. Im Rahmen des vorliegenden Forschungsprojekts war es gelungen ca. 120 Dokumente in Rollenform zu identifizieren. Diese können hier allerdings nicht alle ausführlich vorgestellt werden. Es wurden vielmehr etwa zwei Dutzend besonders repräsentative Exemplare ausgewählt und eingehend vorgestellt (Kapitel 4-6):

Kapitel 4 befasst sich in erster Linie mit Dokumenten aus dem 14. und frühen 15. Jh., die im Städtedreieck zwischen Konya, Tabriz und Bagdad entstanden sein dürften. In einzelnen Fällen dürfte eine Entstehung in Zentralasien im Vordergrund stehen.

Kapitel 5 greift Exemplare auf, die in Iran selbst oder durch Handwerker mit einem iranischen Hintergrund angefertigt worden sind. Sie dürften zumeist aus dem 16.-18. Jh. stammen.
Diese beiden Kapitel (4 und 5) beschreiben die berücksichtigten Exemplare im Rahmen ausführlicher Katalogisate. Sie schenken einerseits ihrer Gestaltung, anderseits ihren textlichen Elementen ausführlich Beachtung. Da die Rollen jeweils sehr individuell gestaltet sind, war es nicht möglich, bei der Beschreibung stets nach demselben Schema vorzugehen. Die an der Herstellung beteiligten Kunsthandwerker und Kopisten haben immer wieder andere Muster gewählt. In andern Fällen stimmt das Layout der einzelnen Rollen zwar weitgehend überein. Da aber die Kopisten beim Ausfüllen der Textbänder und weiterer Figuren auf den jeweiligen Dokumenten wiederum gänzlich anders vorgingen, war auch in diesen Fällen ein völlig unterschiedliches Vorgehen bei der Beschreibung erforderlich.

Die einzige Konstante bei der Beschreibung der Rollen ist wohl die Unterscheidung zwischen Textbändern und Mittelstreifen; diese beiden Elemente lassen sich auf den meisten Belegstücken feststellen. Der Mittelstreifen ist dabei jener Teil der Rolle, der von den beiden seitlichen Schriftbändern, diese sind oft mehrteilig, eingefasst wird.

Kapitel 6 wiederum ist abweichend aufgebaut und umfasst keine eigentlichen Katalogisate. Dies liegt daran, dass in diesem Abschnitt sieben Dokumente aus osmanischen Kontexten (v.a. 17. Jh.) behandelt werden, die bereits in einem frühen Stadium der Untersuchungen als eigenständige Gruppe identifiziert werden konnten. Dies gestattete eine in sich geschlossene Erklärung dieser Belegstücke. Es schien nicht sinnvoll, diese in sich kohärente Argumentation wieder aufzubrechen.

Kapitel 7 bildet den Abschluss dieser Arbeit. Es stellt die späten Entwicklungen in der Tradition der Herstellung derartiger Dokumente in Rollenform vor. Im Vordergrund stehen Belegstücke aus dem 18. und 19. Jh. Sie dürften oft in Indien entstanden sein. Aber auch in Iran wurden während der Qāḡāren-Zeit (1786-1925) weiterhin derartige Dokumente angefertigt. Das jüngste bekannte

(C) TOBIAS NÜNLIST, 2020 | DOI:10.1163/9789004429154_005

This is an open access chapter distributed under the terms of the CC BY-NC-ND 4.o license. 
Exemplar dürfte um 1970 in Syrien hergestellt worden sein (Berlin, Staatsbibliothek, Ms. or. 14101). ${ }^{1}$

Es scheint angezeigt, an dieser Stelle noch einige Punkte speziell festzuhalten: In diesem zweiten Teil mit den Katalogisaten kommen mehrfach Wiederholungen vor. Es wurde bewusst darauf verzichtet, diese Wiederholungen zu beseitigen, dürften doch viele Benutzer gerade im Katalogteil nur einzelne Kapitel konsultieren. Ihnen würden die allenfalls gestrichenen Zusatzinformationen in den Wiederholungen fehlen.

Wichtig scheint auch der Hinweis, dass die Erfassung der auf den Rollen enthaltenen Texte den Erfordernissen kritischer Editionen in keiner Weise genügt. Das Erstellen entsprechender Katalogisate hätte das im Rahmen dieses Projekts Möglichen in zeitlicher und finanzieller Hinsicht gesprengt. Es ist auch klar, dass diese Abschnitte mit den Textstellen auf Arabisch oder Persisch immer noch Fehler enthalten. Es mögen auch zu viele Fehler sein. Allerdings stellt diese Arbeit meines Wissens den ersten Versuch dar, den Inhalt dieser Rollen derart genau vorzustellen. Dies war gerade angesichts des oft schlechten Erhaltungszustands und der vielen verderbten Textstellen kein leichtes Unterfangen. Auch waren oft Schreiber am Werk, deren Kenntnisse des Arabischen bei weitem nicht über alle Zweifel erhaben sind. Die Katalogisate halten die Textstellen grundsätzlich so fest, wie sie auf der Rolle stehen. Dies ist auch dann der Fall, wenn dem Schreiber ganz offensichtlich ein Fehler unterlief. Ein besonderer Dank geht hier an Dr. Edward Badeen (Zürich, Basel), der sich immer wieder Zeit nahm, knifflige Stellen nochmals anzuschauen. Aber dennoch musste vieles offen bleiben.

Es war überhaupt nur Dank modernen technischen Hilfsmitteln möglich, die Dokumente so genau zu erfassen. In vielen Fällen standen von den untersuchten Belegstücken professionell erstellte Digitalisate zur Verfügung. Gerade im Fall der sehr wichtigen Exemplare aus der Chester Beatty

1 Vgl. Kapitel 7.2, bei Anm. 43f.
Library (Dublin) wurden die Beschreibungen aber anhand von eigenen Aufnahmen erstellt, die professionellen Standards in keiner Weise genügen. Die Chester Beatty Library begann mit der Digitalisierung ihrer Rollen erst, als das vorliegende Projekt kurz vor Abschluss stand.

Bei der Identifikation der auf den Rollen kopierten Texte waren die Suchmöglichkeiten des Internets unerlässlich. Hier fanden sich oft erste Hinweise, die eine Bestimmung der einzelnen Texte überhaupt erst ermöglichten. Sehr oft waren es Einträge auf Facebook oder Twitter, in denen sich Gebete desselben Inhalts nachweisen liessen, wie sie bereits auf den Rollen aus dem 14. oder 15 . Jh. enthalten sind. Wo immer möglich wurde versucht, diese Texte in Druckausgaben nachzuweisen. Dies ist oft, aber nicht immer gelungen. Die kopierten Texte liessen sich oft in Gebetssammlungen belegen.

Die in dieser Arbeit zitierten Internetquellen werden oft von Gruppierungen unterschiedlichster religiöser Obedienz betrieben. Der Verweis darauf ist mit keinerlei ideologischen Überzeugungen meinerseites verbunden. Auch bin ich mir bewusst, dass Internetquellen vergänglich sind. Ich betone einfach, dass ich die in dieser Arbeit zitierten Quellen nicht erfunden habe. Bedauerlich ist insbesondere, dass die sehr umfassende Quelle www.knozalasrar.com (für kunūz al-asrār) bereits im Herbst 2017 gänzlich versiegt war.

Die Koranzitate wurden aus der Quelle http:// www.holyquran.net übernommen. Der Wortlaut der gemäss dieser Quelle zitierten Koranstellen stimmt dabei zumeist nicht in allen Details mit der Fassung des Texts überein, wie er auf den einzelnen Rollen steht. Auch wurden die in der angeführten Quelle enthaltene vollständige Vokalisation des Texts belassen; dies bedeutet aber nicht, dass die Stellen auf der Rolle ebenso vokalisiert und mit weiteren Hilfszeichen (šadda, sukūn etc.) versehen sind. Auch hier bestand das eigentliche Ziel darin, aufzuzeigen, dass es sich beim Text auf den Rollen oft um Zitate aus dem Koran handelt. Sie wurden wo immer möglich identifiziert. Aufgrund der häufigen Verwendung der als $\dot{G} u b \bar{a} r$ 
bekannten mikroskopischen Schrift war dies kein leichtes Unterfangen.

Wiederholt wird in den Anmerkungen auch auf Einträge auf Wikipedia verwiesen. Dies trägt mir in Peer review-Verfahren regelmässig sarkastische Bemerkungen und die ultimative Aufforderung ein, wissenschaftlich glaubwürdigere Quellen beizubringen. Diesen Aufforderungen bin ich nicht immer nachgekommen. Es ist festzuhalten, dass auch die Einträge auf Wikipedia heute zumeist einen hohen wissenschaftlichen Standard aufweisen; die aktuelle Situation lässt sich jedenfalls nicht mehr mit jener vor 15 oder 20 Jahren vergleichen. Es kann durchaus sein, dass WikipediaArtikel informativer sind als jene in den einschlägigen islamwissenschaftlichen Referenzwerken. Ausserdem ist hier auf das von Prof. Dr. Patrick Franke (Bamberg) initiierte Projekt hinzuweisen, das darauf abzielt, die Einträge auf Wikipedia zum Islam und zur islamischen Welt in deutscher Sprache fachlich auf den aktuellen Stand der Forschung zu bringen. ${ }^{2}$

Es mag auch sein, dass viele Katalogisate überaus präzis daherkommen und gelegentlich pedantisch wirken. Ich nehme diesen Vorwurf zur Kenntnis. Allerdings ist doch auch dem Umstand Rechenschaft geschuldet, dass vor dieser Untersuchung oft nicht wirklich klar war, was auf diesen Rollen überhaupt steht. Es mag im Nachhinein offensichtlich sein, dass es sich bei den kopierten Texten um Stellen aus dem Koran oder um Gebete handelt. Allerdings war dies nicht von Anfang an selbstverständlich. Nur im Rahmen einer genauen Erfassung war es möglich, die Textstellen zu identifizieren. Es schien mir nicht sinnvoll, die gewonnen Informationen nachträglich wieder zu tilgen.

Es sind hier noch wichtige Hinweise auf die folgenden Punkte anzufügen:

Verwendung einer mikroskopischen Schrift $(\dot{g} u b \bar{a} r)$ : Die untersuchten Exemplare enthalten oft Stellen mit Text in mikroskopischer Schrift,

2 Vgl. https://www.uni-bamberg.de/islamwissenschaft/bie/ biefaqs/ (Stand 26. Januar 2018). auf Arabisch $\dot{g} u b \bar{a} r$ (eigentlich „Staub“) genannt. ${ }^{3}$ Darunter versteht man eine Schrift, deren Höhe zwischen $1-3 \mathrm{~mm}$ beträgt. In der Regel handelt es sich um Nash; grundsätzlich kann aber auch jeder andere Schrifttyp als $\dot{G} u b \bar{r} r$ ausgeführt werden. Diese mikroskopische Schrift ist für die meisten Menschen von blossem Auge nicht zu entziffern. Allerdings darf diese Feststellung nicht darüber hinwegtäuschen, dass es Menschen gibt, die in der Lage sind, Texte in derart kleiner Schrift nicht nur zu lesen, sondern auch zu schreiben. Die vorliegenden Rollen sind dafür Beweis genug. ${ }^{4}$ Derart kleine Schrift wurde oft auch in der Brieftaubenpost eingesetzt, erlaubt sie es doch, möglichst viele Informationen auf geringem Raum unterzubringen. Dokumente aus der Brieftaubenpost aus der islamischen Welt sind allerdings nicht erhalten geblieben.

Bei der Bestimmung der Schrifttypen waren die entsprechenden Übersichten bei N.F. Safwat, D. James und H.A. Faḍāilī immer wieder dienlich. ${ }^{5}$

Bei der Beschreibung geometrischer Figuren wurden runde, mit Text oder Verzierungen ausgefüllte Formen zumeist als Medaillon bezeichnet. Dies geschah in Anlehnung an F. Déroche (1983) sowie R. Würsch, G. Schubert und G. Schoeler. ${ }^{6}$ Es ist allerdings darauf hinzuweisen, dass F. Déroche derartige Figuren in seinen jüngeren Publikationen nicht mehr Medaillons nennt. Der Ausdruck fehlt soweit ersichtlich in seinem

3 Siehe Gacek, Arabic manuscripts 113; Blair, Islamic calligraphy 259-26o (und ihr Index s.v. ghubar); Safwat, Art of the pen 184-192; Vernay-Nouri, Marges 117-131. Auch SourdelThomine et al., Khațț in $E I^{2}$.

4 Auch der Schweizer Schriftsteller Robert Walser (18781956) hat als Mikrogramme bekannte Texte verfasst, die im Rahmen eines Forschungsprojekts neu erschlossen und ediert werden. Beachte: Walser, Mikrogramme 1924-25; Walser Mikrogramme; nach der Transkription von B. Echte und W. Morlang.

5 Safwat, Art of the pen 228-234; James, Qur'äns of the Mamlüks 16-21 („Scripts“); Faḍāilī, Ațlas-i hațt ; siehe auch http:// www.reed.edu/persian-calligraphy/en/ (Stand 12. Januar 2018).

6 Déroche, Manuscrits du Coran Tafel Xxıı und S. 29. Schubert und Würsch, Handschriften 243f. (M III 173; Nr. 101). 
Manuel de codicologie en écriture arabe. ${ }^{7}$ Auch A. Gacek verwendet den Ausdruck nicht. ${ }^{8} \mathrm{M}$. Weisweiler bezeichnet teilweise vergleichbare Figuren auf Bucheinbänden als „Kreise mit Bogenumrahmung". ${ }^{9}$ Da solche Umschreibungen aber allzu komplex erschienen, wurde hier der leichteren Verständlichkeit halber zur Bezeichnung runder, mit Text oder Verzierungen ausgefüllter Figuren der Begriff Medaillon gewählt.

Die Verweise auf das Abbildungsmaterial sind wie folgt $\mathrm{zu}$ verstehen: Die Abkürzung Ав в. (in Grossbuchstaben) verweist auf die in dieser Untersuchung enthaltenen Abbildungen. Die Abkürzung Abb. hingegen wird beim Verweis auf alle andern Illustrationen verwendet; sie stammen zumeist aus Drittquellen (Internetseiten, Bildmaterial aus andern Publikationen etc.). Es ist darauf aufmerksam zu machen, dass sich hochauflösliche Digitalisate der wichtigen Rollen aus der Chester Beatty Library, Dublin, erst beschaffen liessen, als diese Untersuchung weitgehend abgeschlossen war. Die Beschreibung der Belegstücke aus Dublin stützt sich deshalb oft auf eigenes Abbildungsmaterial. ${ }^{10}$

Um die Einordnung der einzelnen Belegstücke zu erleichtern, wurden die wichtigsten Erkenntnisse jeweils am Ende der einzelnen Katalogisate in einem Abschnitt mit der Überschrift Schlussfolgerungen zusammengefasst.

7 Déroche, Abbasid tradition 25 (Tafel 1), bezeichnet runde Verzierungen zur Einteilung von Koranhandschriften zu Gruppen von zehn Versen als „ornamental devices“.

8 Gacek, Arabic manuscripts. A vademecum for readers.

9 Weisweiler, Bucheinband 52; vgl. Déroche, Manuel de codicologie 312-314 (Tafeln 88-90).

10 Es handelt sich um die folgenden Dokumente aus der Chester Beatty Library, Dublin: Is 1624 (Kapitel 4.6); Is 1625 (Kapitel 4.7); Is 1626 (Kapitel 4.9); Is 1623 (Kapitel 5.3); Is 1622 und 1621 (Kapitel 5.5). 